\title{
INSTRUMENTATION FOR HIGH-RESOLUTION STELLAR UV-SPECTROPHOTOMETRY
}

\author{
C. DEJAGER \\ Space Research Laboratory, University of Utrecht, The Netherlands \\ and \\ A. HAMMERSCHLAG and W. WERNER \\ Institute of Applied Physics TNO-TH, Delft, The Netherlands
}

The paper describes the high-resolution stellar UV-spectrometer, experiment S 59 for ESRO TD-1 A satellite. The experiment utilizes the orbit and attitude control characteristics of the TD-1 A to obtain spectra of about $1 \AA$ resolution from a few hundred bright early type stars with an instrument of moderate size. Attitude control of this large three-axis stabilized spacecraft is such that observing-instruments can search the sky along ecliptic meridians which precess by $360^{\circ}$ annum so that complete sky coverage is obtained in half a year.

The experiment consists of a gimballed telescope-spectrometer combination with its own startracking facilities. In the 'stand-by' mode the direction of view follows the search movement of the spacecraft. When a sufficiently bright object is detected in the field of view the mode is switched to 'tracking' whereupon the telescope locks on to the star for a few minutes and performs a spectral scan, while the spacecraft meanwhile continues its search movement. After completion of the measurements the experiment is returned to the stand-by position in the 'reverse' mode.

The accuracy requirements commensurate with the resolution aims are about \pm 1 arc sec in pointing and $\pm 10 \mu \mathrm{m}$ in structural tolerances.

The field of view is $50 \times 10$ arc min with the long side perpendicular to the direction of the search movement. As the precession of the search path is 4 arc min/orbit a minimum of 12 observations per object is obtained. With this arrangement two types of in-flight calibration on celestial objects can be performed.

To reduce size of the instrument as much as possible the spectrometer is folded back into the length of the telescope, which is of the Dall-Kirkham type, by means of an off-axis paraboloidal collimator.

With this arrangement the spectrum is formed out of the plane of symmetry of the instrument, alongside the edge of the primary mirror, from where the light is passed to the detectors below this mirror. In order to obtain sufficient image quality in the spectrum an off-axis paraboloidal-grating is used in a $15 \mathrm{deg}$ off-plane mounting (see Figure 1).

The tracking systems use about $15 \%$ of the collected intensity, which is deflected from the main beam from the image of the primary mirror formed by the secondaryand collimator mirrors. For the 'fine tracking' in the direction of dispersion a linear system is employed. sensing the displacement by means of a vibrating knife edge 


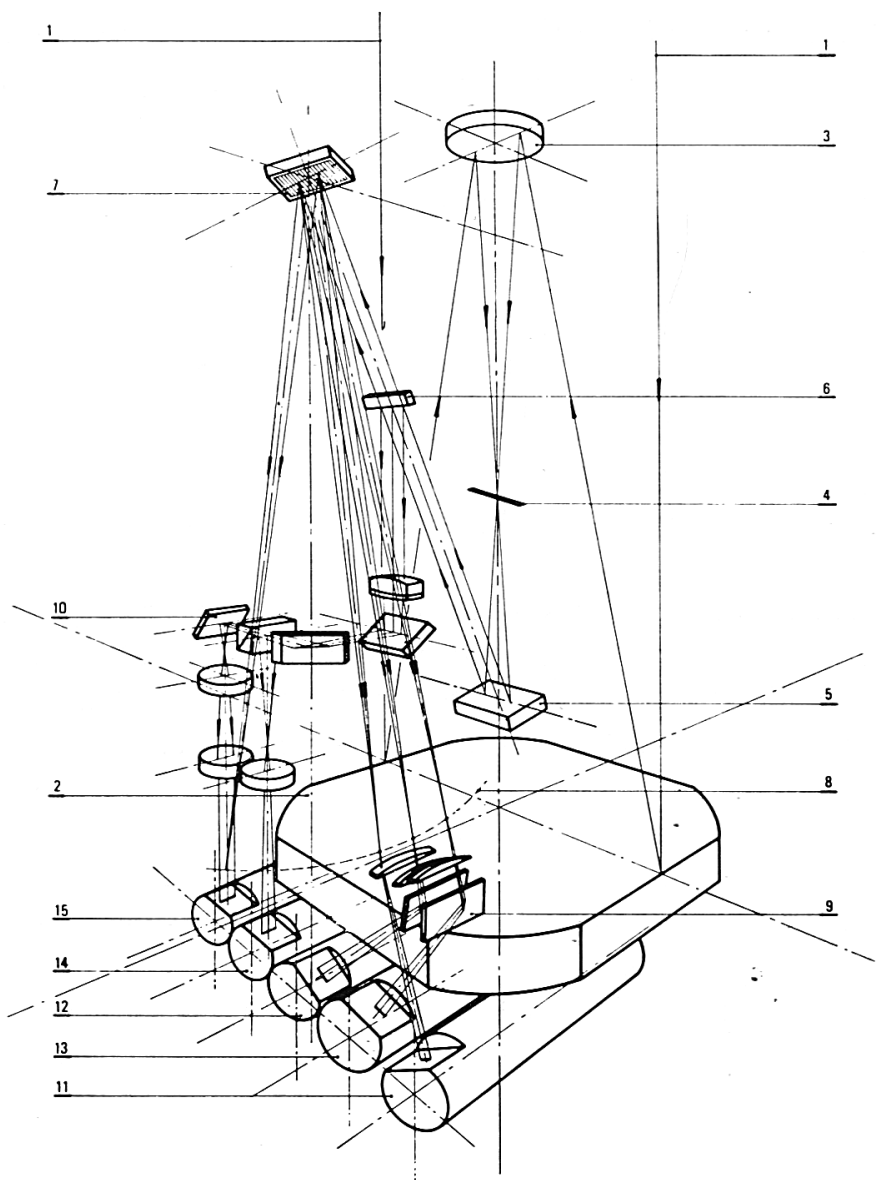

Fig. 1. Optical system of an ultraviolet starspectrometer for the ESRO TD-1 satellite. 1. Incident beam. - 2. Primary mirror (ellipsoid). - 3. Secundary mirror (spherical). - 4. Entrance diaphragm of the spectrometer box. - 5. Collimation mirror. - 6. Small mirror reflecting $10 \%$ of the light for the pointing system. - 7. Off-axis paraboloidal diffraction grating (1200 lines $/ \mathrm{mm})$. - 8. Spectrum. - 9. Condensor system. - 10. Optical elements of the pointing system. $-11,12$ and 13 Photo multipliers for the measurement of the spectrum. -14 and 15 Photo multipliers of the pointing system. The overall length of the instrument is $600 \mathrm{~mm}$. - The main mirror is a square with sides of $220 \mathrm{~mm}$.

chopping through the image formed by the tracker optics. In the perpendicular 'main movement' direction an on-off sensor consisting of a stationary knife edge is used. The actuators for the star-tracking system consist of drive coils in permanent magnetic fields. The spectrum scanner is an optically controlled step-mechanism, employing flexure devices exclusively for the moving parts and a drive system nearly identical to the fine-tracking.

The structural design employs anti-vibration mountings for the complete instrument. The telescope structure consists of a fused silica tripod and an aluminium mirror cell. Fixation of the main mirror is by means of invar spring blades arranged in such a way 
that the suspension is stiff for inertial forces and resilient for thermal expansion and other deformations. Spectrometer and detectordeck are designed as aluminium box structures. The axis of rotation of the fine tracking is defined by a specially designed spring hinge and for the main movement by a lead-lubricated ball bearing. The complete gimbal forms an axis-cross before the primary mirror in the shade of the tripod and the spectrometer box. During launch the moving part is clamped by a pneumatic system.

The electronic system consists of the detectors with high tension and preamplifiers, located in the detectordeck behind the primary mirror, a number of locally situated sensing- and driving amplifiers for the servo-mechanisms and a central electronics box, with the data handling and operational control facilities.

Electrical connections to the moving part are made by means of a flexible printed circuit.

\section{DISCUSSION}

E. B. Jenkins: When is the scheduled launch date for this instrument?

A. Hammerschlag: Scheduled launch: February' 72. 